\title{
Parotid Myoepithelial Carcinoma in a Pediatric Patient with Multiple Recurrences: Case Report
}

\author{
Harold Bravo Thompson ${ }^{a}$ Maria Lim Law ${ }^{b} \quad$ Ruth Vergara Vasquez ${ }^{c}$ \\ Omar Castillo Fernandez ${ }^{b}$ \\ a Medical Doctor, Dr. Arnulfo Arias Madrid Hospital, Panama City, Panama; ${ }^{b} C l i n i c a l$

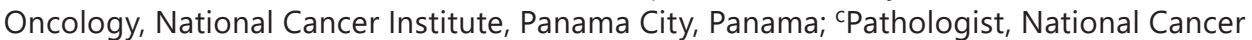 \\ Institute, Panama City, Panama
}

\section{Keywords}

Myoepithelial tumors · Local neoplasm recurrences $\cdot$ Parotid cancer

\begin{abstract}
Myoepithelial carcinoma of the salivary glands is a rare entity, with scarce amount of case reports in the literature. Due to its infrequency, its diagnosis is usually difficult and uncertain. Although there are reports of locoregional recurrences and distant metastases, its low incidence and varied biological behavior limits the clinical evidence that can be used to predict the prognosis and determine the course of treatment. We present a 23-year-old female patient without past medical history with an initial 1-year history of volume increase in the right parotid region of tumor aspect and painful on palpation. As a malignancy was suspected, a total parotidectomy was performed, reporting in the deep lobe a parotid myoepithelial carcinoma with vascular and neural invasion, negative borders, and 3-9 negative regional nodes. During her 16-year clinical evolution, she presented approximately every 2 years and a total of 9 locoregional recurrences and hepatic metastases, including cervical lymphoid nodules, temporal bone, frontal bone, and temporal fossa. Those recurrences have been treated with coordinated efforts between repeated external radiotherapy, chemotherapy, and multiple surgical resections. Myoepithelial tumors represent only $1.0-1.5 \%$ of all salivary gland tumors. The literature reports suggest a high incidence of locoregional recurrences and distant metastases in de novo myoepithelial carcinomas. Due to its rarity, treatment continues to be based on the experience of medical staff.
\end{abstract}

\section{Karger $\stackrel{2}{=}$}




\section{Introduction}

Myoepithelial carcinoma (MC) is a rare neoplasm, almost exclusively composed of tumor cells with myoepithelial differentiation, without evident ductal differentiation and clear tumor infiltration in adjacent tissues [1,2]. Myoepithelial cells are specialized basal cells that occur in the epithelium of various organs, including the salivary glands, respiratory tract, breast, and sweat glands. This proliferation of myoepithelial cells leads to the formation of rare tumors, both benign and malignant [3].

Malignant myoepithelioma represents 1-2\% of all salivary gland neoplasms and $1.2-1.5 \%$ of all carcinomas. It arises mainly from the major salivary glands, most frequently in the parotid gland, and shows an infiltrative growth pattern towards the adjacent tissues $[1,2,4]$. In the pediatric population, salivary gland neoplasms are rare, with an overall incidence of $3-4$ cases per 10,000 , corresponding to $<5 \%$ of salivary gland tumors and $<2 \%$ of head and neck tumors $[5,6]$.

The clinical behavior and outcomes of MC are poorly defined. Although locoregional recurrence and distant metastases rates have been described $[2,3,7,8]$, its low incidence and varied biological behavior limits the clinical utility of the evidence that physicians can use to predict prognosis and make therapeutic decisions. We present a case report of a pediatric patient with multiple local recurrences and ultimately liver metastases from a parotid MC in order to document its histopathological characteristics and therapeutic evolution.

\section{Case Report}

A 7-year-old female patient with no previous medical history presented with a 1-year history of volume increase in the right parotid region which was tumorous, mobile, and had progressively painful appearance, not accompanied by constitutional syndrome. At physical exam, a right parotid mass $\sim 5 \mathrm{~cm}$ in diameter stands out, with a hard consistency, mobile, but attached to deep planes, and elicits pain on palpation. Due to high suspicion of a malignancy, she underwent surgery for total parotidectomy plus cervical dissection. Pathology reported parotid MC with vascular and neural invasion and negative margins in the deep lobe of the parotid, 3-9 negative regional nodes (shown in Fig. 1, 2).

One year later, the patient sought medical attention for a new increase in volume in the same parotid region. Biopsy was done and reported $\mathrm{MC}$, together with immunohistochemical analysis (IHC): CKAE1/AE3 positive, calponin positive, S-100 negative, and SMA negative (shown in Fig. 3). She was taken to the operating room again for surgical excision with enlarged margins including right mandibular ramus, submandibular nodes, and right infraauricular nodes. Afterwards, it was reported as MC in the lymph node group.

Postsurgical control CT reported a $5.1 \times 4.5 \mathrm{~cm}$ mass that occupies the right infratemporal fossa and compresses the parapharyngeal space, potentially associated with the residual tumor. She was then sent to palliative radiotherapy (50 Gy in 25 fractions) in the right temporal fossa for locally advanced residual disease (age 9 years).

Three months after the end of radiotherapy, she presented the third recurrence localized in the right temporal region that eroded the external and internal bone cortex and was $1.5 \mathrm{~cm}$ in diameter, with a histopathological report of metastatic MC. The patient was reirradiated in the right temporal fossa 5 months after the first radiation (45-50 Gy in 25 fractions); 2 months after the end of radiotherapy, she was treated with 10 cycles of cyclophosphamide/doxorubicin/cisplatin.

She continued follow-up by images, and 3 years later (12 years old), the fourth recurrence was found as an extradural mass in the right temporal fossa. Palliative radiotherapy 


\section{Case Reports in Oncology}

\begin{tabular}{l|l}
\hline Case Rep Oncol 2021;14:989-997 \\
\hline DOI: 10.1159/000515783 & $\begin{array}{l}\text { @ 2021 The Author(s). Published by S. Karger AG, Basel } \\
\text { www.karger.com/cro }\end{array}$ \\
\hline
\end{tabular}

Bravo Thompson et al.: Parotid Myoepithelial Carcinoma in Pediatrics

Fig. 1. Architectural view of parotid myoepithelial carcinoma with a nodular pattern of infiltration into adjacent tissues. Hematoxylin-eosin $\times 1.1$.
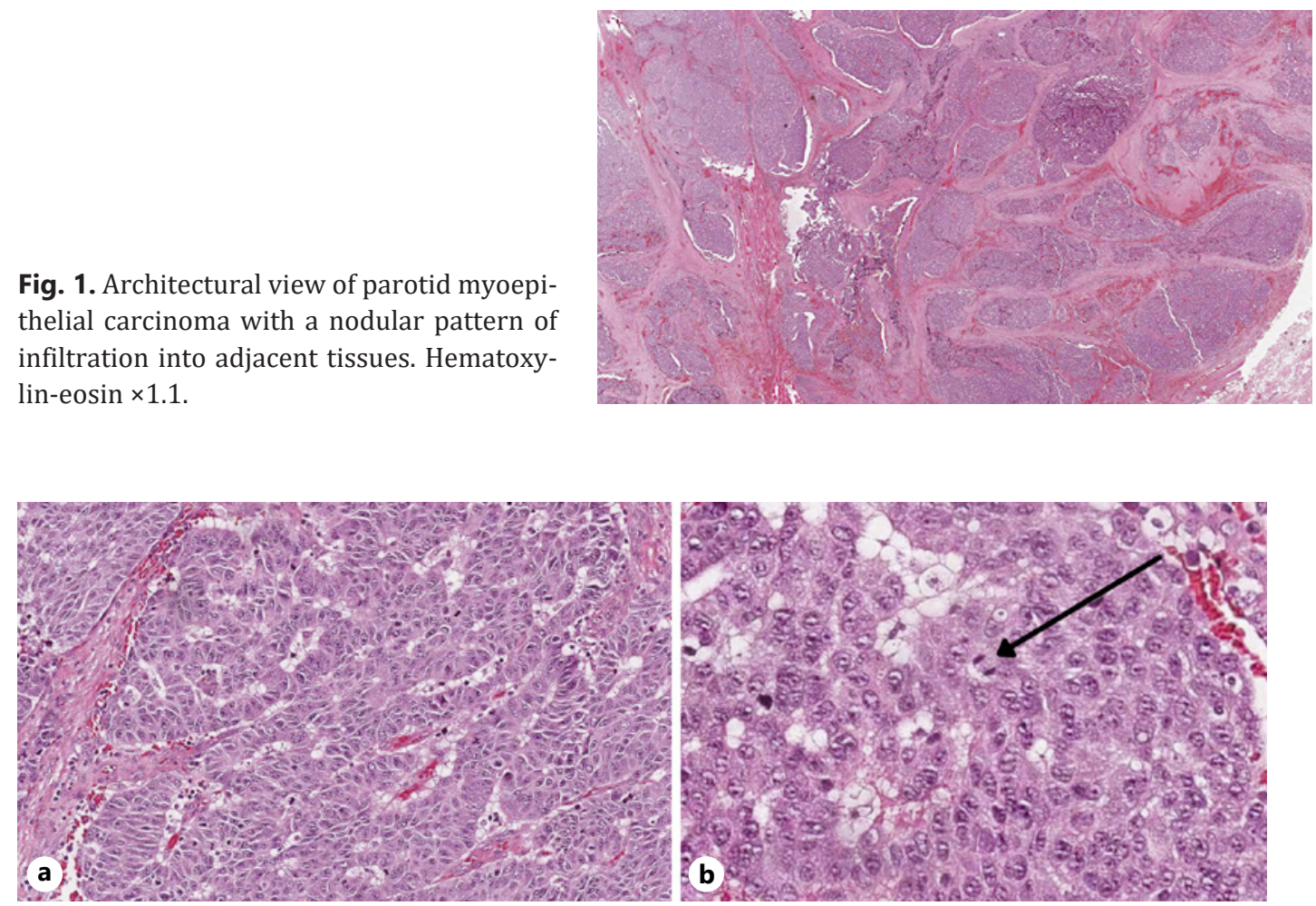

Fig. 2. Cytological pattern of parotid myoepithelial carcinoma. Hematoxylin-eosin $\times 20$. a Epithelioid-like cells with trabecular pattern. b An epithelial cell pattern, clear and eosinophilic cytoplasm, moderate nuclear pleomorphism with mitosis (black arrow) is observed (original magnification $\times 20$ ).

was considered; however, it was ruled out since the right eye and the brain had reached the tolerance limit to irradiation. She was treated with 9 cycles of cyclophosphamide/doxorubicin/cisplatin.

During follow-up 3 years later (15 years old), she presented the fifth recurrence in the frontal bone, which was resected surgically. Two years later (17 years old), she presented the sixth recurrence as a 2-cm level IIB right cervical lymphoid nodule. The decision was to perform a selective IIB neck dissection + levels III and IV examination. Afterwards, the surveillance strategy was chosen, given the lack of evidence of adjuvant treatment in this setting, in addition to having received a maximum dose of doxorubicin.

However, 2 months later, she presented with a $5 \times 8 \mathrm{~cm}$ obvious lesion in the posterior triangle of the neck that crosses the midline consistent with the seventh recurrence of MC. She was not a candidate for surgery or radiotherapy, so 6 cycles of carboplatin/paclitaxel were started.

She evolved with complete response in images until 9 months later (18 years old) when the eighth recurrence of MC was evident as a movable lesion with a smooth and rounded surface of $2.5 \mathrm{~cm}$ on the trapezius, which was resected with negative margins. Additionally, she received for the third time radiotherapy to the right neck 40 Gy in 20 fractions. Subsequently, for 40 months, no tumor activity was evidenced, but then the ninth recurrence of the disease presented as a 1.2-cm hypodense heterogeneous nodule in the dorsal area to the distal right internal jugular (level C3) associated with multiple round hepatic lesions in IVA and VIII segments, with hypodense center and peripheral enhancement. Subsequent biopsy reveals poorly differentiated neoplasia with IHC that supports MC. 


\section{Case Reports in Oncology}

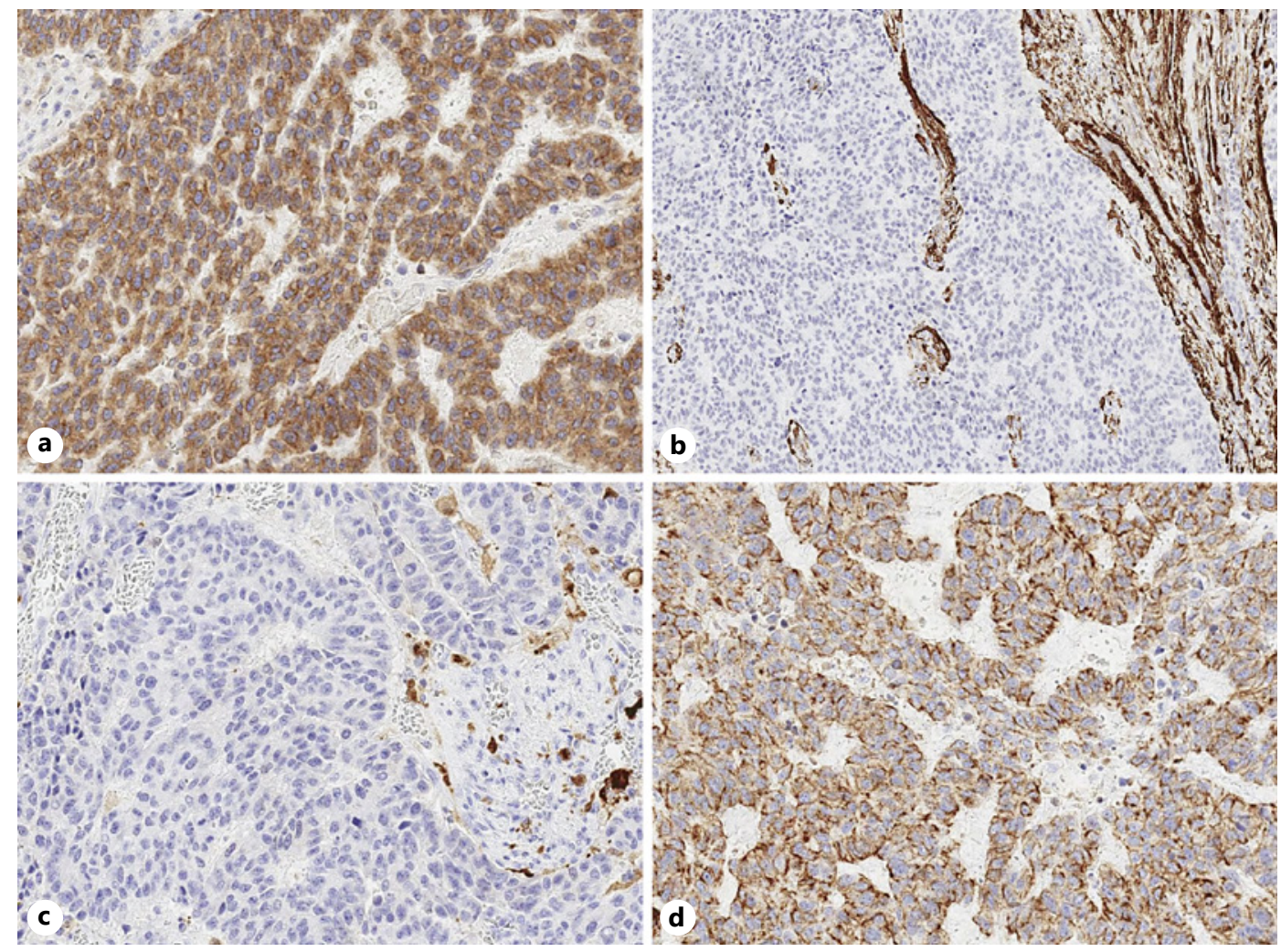

Fig. 3. Immunohistochemistry staining. a CKAE1/AE3 positive. $\times 20$. b Smooth muscle actin (SMA) negative. $\times 20$. c S-100 negative. $\times 20$. d Calponin positive. $\times 20$.

Due to the need to rethink new therapeutic strategies after numerous recurrences, molecular sequencing tests (Foundation One ${ }^{\circledR} \mathrm{CDx}$ ) found a tumor mutational load of 11 muts/Mb, and a C34R alteration in the CTNNB1 gene was discovered. The current treatment plan at the time of documentation (23 years old) consists of re-exposure to 8 cycles of carboplatin/paclitaxel with doses adjusted according to liver function.

\section{Discussion}

\section{Epidemiology}

Parotid MC accounts for $<1-2 \%$ of salivary gland tumors in adults $[2,9,10]$. Up to the year 2015, only 150 cases of MC had been reported in the literature $[9,11]$. However, this tumor is potentially underrecognized, as described by a larger clinical series using the National Cancer Database (NCDB) in the USA [4]. Due to increasing case reports, malignant myoepithelioma was included in the second edition of the World Health Organization histological classification of salivary gland tumors $[2,4]$.

These tumors occur in both sexes, with some reports of slight female predominance. They may occur at any age, but more frequently in the 5th-6th decades of life (range 14-77 years; mean 61.7 years) $[1,2,4]$. On the other hand, in the pediatric population, the real frequency of these tumors is unknown; however, the same proportion of benign and malignant myoepitheliomas has been suggested $[12,13]$. Median age has been reported at 16 years. This case report adds to the few reported cases of MC in children [5]. Compared to their adult 
counterpart, these tumors have a greater predisposition to be malignant, but associated with a more favorable prognosis $[5,14,15]$.

\section{Etiology}

The rarity of this tumor could explain the lack of knowledge about risk factors and pathophysiology in the development of MC. Ionizing radiation from bombs or radiotherapy has been associated with the development of salivary gland neoplasms, and endogenous hormones together with genetic factors have only been associated with rare cases of acinic cell carcinoma [13]. In this case report, the patient did not show any apparent risk factor.

Considering molecular risks factors, exon 3 of the CTNNB1 gene encodes part of the $\mathrm{N}$-terminal domain of the $\beta$-catenin protein, a key phosphorylation site. Genetic mutations that lead to constitutive activation of the Wnt/ $\beta$-catenin pathway have been described as early events in the development of cancer, seen in colorectal cancer, melanoma, cervical cancer, and hepatoblastoma, among others [16]. Pentheroudakis et al. [17] used samples from primary tumors of unknown origin and found that $20.7 \%$ of them exhibited mutations in exon 3/4 of the CTNNB1 gene. This case report included a positive CTNNB1 exon 3 mutation which may have contributed to the development of this rare tumor or its several recurrences, but more studies are needed to assert this.

\section{Clinical Presentation}

Most MCs develop secondary to a pleomorphic adenoma or a preexisting benign myoepithelioma, generating a low-grade neoplasm. When they appear de novo, as in our case, they are usually high grade with a tendency towards clinical and radiological aggressiveness, in addition to a short evolution time $[1,9,10,12]$. A large clinical series reports that $75 \%$ of the MCs appear as "de novo" and 25\% come from a previous benign tumor; other authors describe $50: 50$ ratios and even as low as $30 \%$ de novo MC $[2,7,9]$.

The parotid gland (29-82\%) and the palate (21\%) are the most frequent locations of myoepithelioma in the head and neck region $[2,4,9]$. MCs have also been reported in a variety of sites: jugal, gingival, buccal, nasopharyngeal, laryngeal, pharyngeal and parapharyngeal spaces, nasal cavity, alveolus, retromolar area, base of tongue, cavernous sinus, and maxillary sinus $[9,10]$. Also, MCs outside the head and neck have been reported in the ribs, kidney, bladder lacrimal gland, ocular conjunctiva, infratemporal fossa (as one of the recurrences in our case), lung, trachea, skin, breast, and vulva [3, 7, 9, 14,15]. Metastases have been reported up to 43 years after diagnosis, especially in those secondary to pleomorphic adenoma [9], and in this case report, we can identify the appearance of liver metastases 16 years after the initial presentation.

MC usually presents as a painless swelling, but more frequently with additional symptoms compared to benign ones such as long evolution, rapid growth, pain (10-29\%), facial paralysis (10-15\%), numbness of the tongue or lips, dysphagia, stone consistency, fixation to deep structures, and pathological lymphoid nodules $[1,9,10]$. In our case, the patient presented with an increase in the volume of the parotid region that was painful and fixated to deep structures, which strongly oriented towards a malignant character. The duration of symptoms before diagnosis can vary from 1 month to 3 years (in our case, 1 year). Some hypotheses include that the tumor remains small for a time and then grows rapidly [1].

\section{Diagnostic Methods}

Imaging studies such as nuclear MRI, CT, and PET/CT are helpful to determine tumor extension, as well as in identifying metastases and recurrences $[1,12]$. However, MRI delineates salivary gland tumors more clearly and is useful in detecting facial nerve compromise,

\section{Karger's}


so it is considered the optimal imaging modality $[9,10]$. The final diagnosis is given by the pathologist with a tissue biopsy [9].

\section{Histology and Differential Diagnosis}

Grossly, MC presents as well-circumscribed, uncapsulated tumors, with a nodular or lobed appearance. The cut surface is usually gray to tan white, shiny, myxoid, or gelatinous $[2,14]$. Microscopically, MC is characterized by histological heterogeneity, with various cellular morphologies (epithelioid 29\%, plasmacytoid 14\%, spindle $12 \%$, stellate $16 \%$, and mixed 24\%) and different architectural patterns (multinodular, nests, sheet, and laces) frequently coexisting. Cells have thick or vesicular chromatin, prominent and often enlarged nucleoli, and nuclear pleomorphism with numerous mitoses. An abundant hyaline and/or myxoid matrix with focal metaplastic changes can be observed. Vascular or neural invasion may be present $[1-3,9,12,14]$. This case reports epithelioid cells with a multinodular pattern.

Due to its varied presentation, IHC is required for confirmation including expression of cytokeratins (CKAE1/AE3 100\%), epithelial membrane antigen (21-27\%), calponin (75-100\%), and SMA (35-64\%). Other markers include S-100 protein (41-100\%), vimentin (100\%), glial fibrillary acidic protein (31\%), CAM 5.2 (89\%), and p63 (87.5\%) [1, 2, 10-12]. In our case, the tumor cells are positive for pancytokeratin and calponin, but negative for SMA, S-100, and epithelial membrane antigen. Calponin and vimentin are particularly sensitive for myoepithelial differentiation in salivary gland tumors, and together with S-100 should be included in IHC panels $[2,11]$.

The currently accepted diagnostic criteria include exclusive cytological myoepithelial differentiation (morphology and IHC) and its malignancy with invasion of the salivary glands and neighboring tissues. At the molecular level, several genetic events have been reported: PLAG1 rearrangements (53\%), TGFBR3-PLAG1 fusion (15\%), and HMGA2 (10\%) [2].

Numerous malignancies are included in the differential diagnosis of MC, depending on the predominant cell type (shown in Table 1). Those of epithelioid predominance are relevant, as they can be confused with other salivary gland neoplasms, especially adenoid cystic carcinoma or epi-MC [2].

\section{Treatment and Prognosis}

Although there are no clear guidelines for the specific treatment of MC, for localized salivary gland malignant tumors, wide margins surgery is accepted as the most effective modality $[1,8,12]$. Lymph node dissection can be considered in cases of lymph node metastasis confirmed by physical examination and/or imaging studies. Adjuvant radiotherapy is also commonly considered; however, the precise indications are controversial yet $[1,8]$.

Unlike their benign counterpart, MCs are associated with an increased frequency of local recurrences and metastasis (up to one-third), which requires close clinical follow-up. Adjuvant chemotherapy can help prevent metastasis or recurrences, but its value is not fully established, and some authors suggest that adjuvant chemotherapy does not significantly improve the prognosis of patients $[2,8,10]$.

Previously, MC was considered a low-grade carcinoma, with a low tendency for local recurrences and metastasis. However, studies have reported $6.3-41 \%$ of patients developing nodal metastasis, a $40-47 \%$ distant metastasis rate, and a $46.1-51.9 \%$ recurrence rate. The overall survival rates reported were $78 \%$ at 2 years and $71-78 \%$ at 5 years $[2,4,8]$.

Zheng et al. [8] proposed interstitial brachytherapy with radioactive $\mathrm{I}^{125}$ to improve local control of the MC in patients with recurrent or advanced disease and concluded that it could be considered an effective modality in these types of patients. In those patients with primary disease, they reported a 5-year relapse-free survival of $100 \%$, while in those with recurrent disease, a 5-year relapse-free survival of $25.3 \%$ [8].

\section{Karger'}


Table 1. Histological differential diagnosis

Epithelioid cells

Luminal cells and tubular structures
Adenoid cystic carcinoma

Low-grade polymorphic adenocarcinoma

Epi-MC

Adenocarcinoma NOS

Adenoid cystic carcinoma

Epi-MC

Adenocarcinoma

Plasmacytoid cells

Spindle cells

Clear cells
Plasmacytomas

Melanomas

Nerve sheath tumors

Leiomyosarcomas

Synovial sarcomas

Fibrohistiocytomas

Fibrosarcomas

Sarcomatoid squamous cell carcinomas

Clear cell carcinoma NOS

Epi-MC

Mucoepidermoid carcinoma

Sebaceous carcinoma

Acinic cell carcinoma

Metastasis of a renal carcinoma

Adapted from Bascones A, Escribano A, de la Sen O, Falahat F, Martín-Granizo R, Berguer A. Carcinoma mioepitelial de glándula submaxilar. Caso clínico y revisión de la literatura. Rev Esp Cir Oral y Maxilofac. 2015;37(4):233-8.

NOS, not otherwise specified; MC, myoepithelial carcinoma.

In this case report, the patient presented multiple local recurrences despite efforts in different therapeutic modalities for local control and the last time with evidence of distant metastases. The patient in the case was managed with several surgical procedures with extended margins, radiotherapy, and multiple cycles of systemic chemotherapy, achieving complete clinical and radiological response at the time of documentation but with subsequent recurrence and appearance of distant metastases. Thus, the multiple recurrences and the ability to metastasize demonstrate aggressiveness and resistance in these types of tumors as documented.

Kane et al. [11] in their clinical series found a mean disease-free survival of 31.9 months. Additionally, they associated the following factors with a high probability of metastasis: tumors with $>50 \%$ necrosis, positive margins, high mitotic index ( $>4$ per $10 \mathrm{HPF}$ ), a Ki-67 index between 4 and 10\%, cellular atypia, and spindle-cell pattern [11]. Jiang et al. [18] concluded that recurrence and metastasis have a positive Ki-67 and p53 risk factor and the worst prognosis with p632 overexpression; however, other authors suggest that the expression of p53 is not useful for assessing the risk of recurrence [12]. Regarding tumor recurrence, it has been correlated with the type of tumor cells (63\% stellar morphology or pleomorphism), overexpression of p63, the existence of bone or perineural invasion, and tumor size $(>6 \mathrm{~cm})[8,9,11]$. 
Finally, regarding follow-up, Bascones et al. [1] suggested that correct patient follow-up encompasses a minimum period of 24 months. In our patient, close follow-up was maintained throughout her 9 recurrences and at the time of continuous documentation with her clinical follow-up.

\section{Statement of Ethics}

This case report complies with international guidelines for human studies based on World Medical Association Declaration of Helsinki and further addendum. The authors declare that the patient in this case report provided written informed consent to publish this case, including histological images depicted.

\section{Conflict of Interest Statement}

The authors have no conflicts of interest to declare.

\section{Funding Sources}

Roche Pharmaceuticals assumed the costs of molecular diagnostic test for this case report, which was performed with Foundation One ${ }^{\circledR} \mathrm{CDx}$.

\section{Author Contributions}

Bravo H.: concept and design, acquisition of data, drafting of the manuscript, and review of the literature. Lim M.: concept and design, acquisition of data, critical revision of the manuscript for intellectual content, and aims. Vergara R.: acquisition of histological figures and critical revision of the manuscript for intellectual content. Castillo O.: acquisition of data and critical revision of the manuscript for intellectual content. All authors approved the final version of the manuscript.

\section{References}

1 Encinas Bascones A, Escribano Adam N, de la Sen O, Falahat F, Martín-Granizo R, Berguer A. Carcinoma mioepitelial de glándula submaxilar. Caso clínico y revisión de la literatura. Rev Esp Cir Oral y Maxilofac. 2015;37(4): 233-8.

2 Xu B, Katabi N. Myoepithelial carcinoma. Surg Pathol Clin. 2021 Mar;14(1):67-73.

3 Yokose C, Asai J, Kan S, Nomiyama T, Takenaka H, Konishi E, et al. Myoepithelial carcinoma on the right shoulder: case report with published work review. J Dermatol. 2016;43(9):1083-7.

4 Xiao CC, Baker AB, White-Gilbertson SJ, Day TA. Prognostic factors in myoepithelial carcinoma of the major salivary glands. Otolaryngol Head Neck Surg. 2016 Jun;154(6):1047-53.

$5 \mathrm{Xu}$ B, Aneja A, Ghossein R, Katabi N. Salivary gland epithelial neoplasms in pediatric population: a singleinstitute experience with a focus on the histologic spectrum and clinical outcome. Hum Pathol. 2017;67: 37-44.

6 Calderón Elvir C, Francisco J, Zamora G, Manuel J, Aguilar R. Tumores mioepiteliales de glándulas salivales en pediatría. Acta Pediatr Mex. 2001;22(5):361-7.

7 Wang Z, Herrington B, Schwartz M, Laucirica R. Myoepithelial carcinoma of the parotid gland metastatic to the kidney: case report and review of the literature. Diagn Cytopathol. 2009;38(4):279-82.

8 Zheng L, Lv X, Shi Y, Zhang Y, Yu G, Zhang J. (125)I interstitial brachytherapy for the treatment of myoepithelial carcinoma of the oral and maxillofacial region. Brachytherapy. 2016;15(2):240-5. 
9 Vilar-González S, Bradley K, Rico-Pérez J, Vogiatzis P, Golka D, Nigam A, et al. Salivary gland myoepithelial carcinoma. Clin Transl Oncol. 2015;17(11):847-55.

10 Pandey R, Biswas R, Ray M, Ramteke PP, Dhamija E, Halder A. Report of a unique case of myoepithelial carcinoma of left parotid gland with metachronous bilateral cavernous sinus metastasis. J Egypt Natl Canc Inst. 2018;30(2):73-6.

11 Kane SV, Bagwan IN. Myoepithelial carcinoma of the salivary glands: a clinicopathologic study of 51 cases in a tertiary cancer center. Arch Otolaryngol Head Neck Surg. 2010;136:702-12.

12 García-Ortega F, Carcasés Ortiz M, Martínez Reig S, Beiva González M, Duran R, Malluguiza Calvo J. (Salivary gland myoepithelioma). Acta Otorrinolaringol Esp. 2001;52(3):269-72.

13 Santos KCP, Matsuzaki H, Unetsubo T, Tsuyoshi S, Nagatsuka H, Asaumi JI. De novo myoepithelial carcinoma with multiple metastases arising from a submandibular salivary gland: a case report. Oncol Lett. 2017;13(4): 2679-83.

14 Biradar P, Menon S, Patil A, Karimundakal G, Jambhekar N. Primary myoepithelial carcinoma of rib bone: morphology, immunohistochemical evaluation and diagnostic dilemma in an unusual case. J Cancer Res Ther. 2015;11:647.

15 Khademi B, Kazemi T, Bayat A, Bahranifard H, Daneshbod Y, Mohammadianpanah M. Salivary gland myoepithelial neoplasms: a clinical and cytopathologic study of 15 cases and review of the literature. Acta Cytol. 2010; 54(6):1111-7.

16 Gao C, Wang Y, Broaddus R, Sun L, Xue F, Zhang W. Exon 3 mutations of CTNNB1 drive tumorigenesis: a review. Oncotarget. 2018;9(4):5492-508.

17 Pentheroudakis G, Kotteas EA, Kotoula V, Papadopoulou K, Charalambous E, Cervantes A, et al. Mutational profiling of the RAS, PI3K, MET and b-catenin pathways in cancer of unknown primary: a retrospective study of the hellenic cooperative oncology group. Clin Exp Metastasis. 2014;31:761-9.

18 Jiang YH, Cheng B, Ge MH, Zhang G. The prognostic significance of p63 and Ki-67 expression in myoepithelial carcinoma. Head Neck Oncol. 2012;4:9-21. 